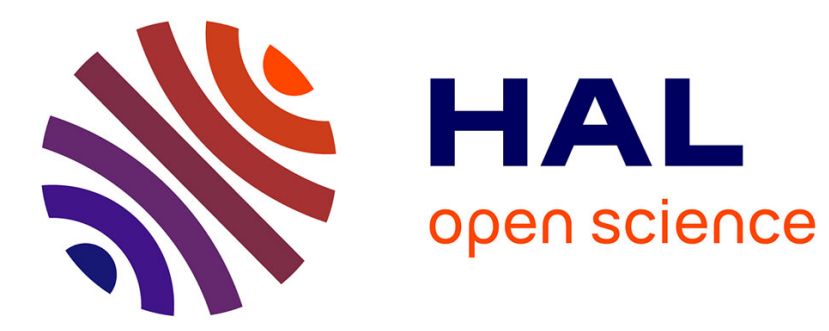

\title{
Solar astrophysical fundamental parameters
}

\author{
Mustapha Meftah, Abdanour Irbah, Alain Hauchecorne
}

\section{To cite this version:}

Mustapha Meftah, Abdanour Irbah, Alain Hauchecorne. Solar astrophysical fundamental parameters. Proc. SPIE 9143, Space Telescopes and Instrumentation 2014: Optical, Infrared, and Millimeter Wave, Jun 2014, Montréal, Canada. pp.91431R, 10.1117/12.2050136 . hal-01070129

\section{HAL Id: hal-01070129 \\ https://hal.science/hal-01070129}

Submitted on 30 Sep 2014

HAL is a multi-disciplinary open access archive for the deposit and dissemination of scientific research documents, whether they are published or not. The documents may come from teaching and research institutions in France or abroad, or from public or private research centers.
L'archive ouverte pluridisciplinaire HAL, est destinée au dépôt et à la diffusion de documents scientifiques de niveau recherche, publiés ou non, émanant des établissements d'enseignement et de recherche français ou étrangers, des laboratoires publics ou privés. 


\title{
Solar astrophysical fundamental parameters
}

\author{
M. $\operatorname{Meftah}^{a,^{*}}$, A. $\operatorname{Irbah}^{a}$, A. Hauchecorne ${ }^{a}$ and the PICARD team \\ ${ }^{a}$ Université Versailles St-Quentin; Sorbonne Universités, UPMC Univ. Paris 06; CNRS/INSU, \\ LATMOS-IPSL, 11 Boulevard d'Alembert, 78280 Guyancourt, France
}

\begin{abstract}
The accurate determination of the solar photospheric radius has been an important problem in astronomy for many centuries. From the measurements made by the PICARD spacecraft during the transit of Venus in 2012, we obtained a solar radius of $696,156 \pm 145$ kilometres. This value is consistent with recent measurements carried out atmosphere. This observation leads us to propose a change of the canonical value obtained by Arthur Auwers in 1891. An accurate value for total solar irradiance (TSI) is crucial for the Sun-Earth connection, and represents another solar astrophysical fundamental parameter. Based on measurements collected from different space instruments over the past 35 years, the absolute value of the TSI, representative of a quiet Sun, has gradually decreased from $1,371 \mathrm{~W} \cdot \mathrm{m}^{-2}$ in 1978 to around $1,362 \mathrm{~W} \cdot \mathrm{m}^{-2}$ in 2013 , mainly due to the radiometers calibration differences. Based on the PICARD data and in agreement with Total Irradiance Monitor measurements, we predicted the TSI input at the top of the Earth's atmosphere at a distance of one astronomical unit $(149,597,870$ kilometres $)$ from the Sun to be $1,362 \pm 2.4 \mathrm{~W} \cdot \mathrm{m}^{-2}$, which may be proposed as a reference value. To conclude, from the measurements made by the PICARD spacecraft, we obtained a solar photospheric equator-to-pole radius difference value of $5.9 \pm 0.5$ kilometres. This value is consistent with measurements made by different space instruments, and can be given as a reference value.
\end{abstract}

Keywords: solar radius, total solar irradiance, solar oblateness, space instrumentation, telescope, radiometer.

\section{INTRODUCTION}

Measurements of the solar radius are of great interest within the scope of the debate on the role of the Sun in climate change. Solar radius is mainly related to the knowledge of the solar atmosphere. However, it is very difficult to measure this fundamental parameter of astrophysical interest. In addition, total solar irradiance (TSI) is a crucial input for all climate models. In closing, solar equator-to-pole radius difference $\left(\Delta r=R_{e q}-R_{p o l}\right)$ is a fundamental property of our star and its knowledge will enrich the coming dynamical models of Sun and stars. The solar oblateness $\left(\Delta_{\odot}\right)$ corresponds to the excess ratio of the equatorial solar radius $\left(R_{e q}\right)$ to the polar radius $\left(R_{\text {pol }}\right)$, which is of great interest for those working in relativity and different areas of solar physics. The solar equator-to-pole radius difference is another solar astrophysical fundamental parameters, which is difficult to determine and requires a specific attention. These three essential parameters are still under research with respect to their absolute determination. This finding sheds new light on these fundamental parameters of solar physics.

PICARD is a mission dedicated to the simultaneous measurements of the total and spectral solar irradiance's, its diameter and asphericity. It also probes seismically the solar interior by analysing its local intensity variation. PICARD contains a double program with in-space and on-ground measurements (see Figure 1). Space observations are a priori most favourable, however, space entails also technical challenges, a harsh environment, and a finite mission lifetime. The PICARD spacecraft was launched on June 15, 2010 and was retired in April 2014. On ground, the instruments are less affected by in-space degradation and maintenance is easily provided so if the atmosphere is properly monitored and taken into account, they still represent an opportunity to generate the needed long-term time-series of solar radius. That is why ground measurements have been carried out since May 2011-and is currently being pursued despite the end of the space program. In this paper, we present our current results. In particular, we show new estimates of the absolute values of the solar radius, the total solar irradiance, and the solar oblateness.

\footnotetext{
* Corresponding author

E-mail address: Mustapha.Meftah@latmos.ipsl.fr
} 

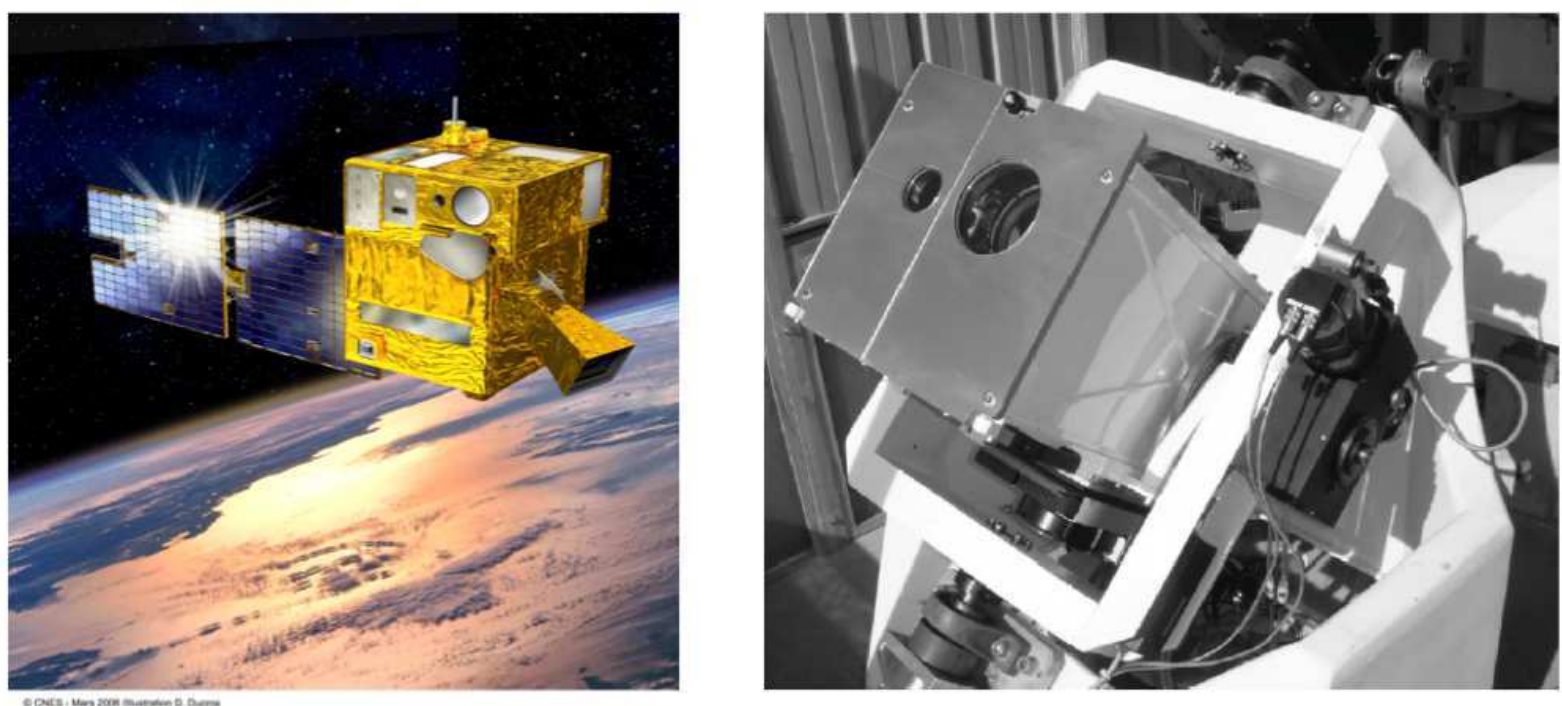

Figure 1. (left) PICARD is a spacecraft dedicated to simultaneous measurement of the solar radius, the solar shape, the solar irradiance and the solar interior. The payload consists of two absolute radiometers (provided by Royal Meteorological Institute of Belgium (RMIB) and by Physikalisch Meteorologisches Observatorium of Davos (PMOD)) measuring the TSI, and a Ritchey-Chrétien imaging telescope to determine the solar radius and asphericity (LATMOS). (right) "PICARD SOL" (under the responsibility of Observatoire de la Côte d'Azur (OCA) and LATMOS) is a set of instruments based at the Calern site (Observatoire de la Côte d'Azur, France) to carry out solar radius with the capability in discriminating as much as possible the measurements affected by atmospheric effects. A replica of the space instrument was installed at the Calern observatory, which provides a nearly continuous record of the solar radius at different wavelengths since May 2011. The picture corresponds to the telescope equatorial mount. The replica of the space telescope is placed in a vacuum tank closed by a glass window.

\section{SOLAR RADIUS}

Solar radius determination is one of the oldest problems in astrophysics. Jean Picard (1620-1682), a pioneer of precise modern astrometry, made regular observations of the Sun. Measurements made during the seventeenth century were then patiently rediscovered and studied by Elizabeth Nesme-Ribes. ${ }^{1}$ Michel Toulmonde re-analysed these measurements ${ }^{2}$ and his investigations reveal the need for instrumental corrections (atmospheric refraction, seeing, diffraction, etc.). Thus this analysis gives a mean solar radius at one astronomical unit (AU) of 959.9 arcseconds in 1670 (around the deep Maunder minimum).

At the end of the nineteenth century, an investigation of the value of the solar radius, obtained by meridian observations, was carried out by Arthur Auwers. He published a value for the solar radius of $959.63 \operatorname{arc}^{-s e c o n d s}{ }^{3}$ (arbitrary value resulting from discussion of visual observations), where a large correction for the irradiation effects was introduced. In solar modelling, this canonical value has been commonly used and was adopted by the International Astronomical Union (IAU). Solar models are calibrated to this photospheric radius, defined by the point in the atmosphere where the temperature equals the effective temperature, by adjusting some measurements of convective efficacy, such as the mixing length. Measuring the diameter of a planet (Mercury for example) or of the Moon has no ambiguity with respect to the measurement because the edge of the body being studied is solid in nature. Measuring the diameter of the Sun (gaseous sphere) remains complex. The diameter of the Sun is based on an arbitrary definition because of the gaseous nature of our star. Different instruments have been used to measure the solar radius and the set of the values obtained shows a lack of coherence. The main reasons for these differences are also related to the nature of the instruments (point spread function, resolution, distortion, plate scale, etc.), the spectral domain of observation, and instrumental effects (atmospheric effects for ground instrument and harsh space environment for flight instrument). In general, the solar radius is obtained from each image in several solar position angle sectors from the solar limb darkening function. The solar radius is usually measured by determining the inflection point of solar limbs (conventional method). 
The transit of Venus on June 2012 provided a unique opportunity to determine the radius of the Sun using solar imagers. The transit was observed from space by the PICARD spacecraft. From this, the value of the solar radius from one $\mathrm{AU}$ was found to be equal to 959.86 arc-seconds at $607.1 \mathrm{~nm}^{4}$ using the conventional method, whereas the estimated uncertainties of the measurements, random plus systematic, are typically less than 0.2 arcsecond. The PICARD solar photospheric radius is higher by about 0.23 arc-second than the normally used value of 959.63 arc-seconds $^{5}$ (see Figure 2). The Michelson Doppler Imager (MDI) aboard the Solar and Heliospheric Observatory (SoHO) observed the transits of Mercury on 2003 and 2006. ${ }^{6}$ This instrument also gives a value of 0.49 arc-second more than that commonly used. Recent measurements made by the Solar Disk Sextant (SDS) instrument provide a solar radius close to 959.856 arc-seconds $^{7}$ at $615 \mathrm{~nm}$. This result is similar to that found by the instrument of the PICARD mission. To further consolidate the PICARD result, a new method has been developed (definition of the solar radius other than the inflection point) to estimate the solar radius during the transit of a planet in front of the Sun. ${ }^{8}$ It is based on the estimation of the spectral solar radiance decrease in a region around the contact between the planet and the Sun at the beginning of the ingress and at the end of the egress. The extrapolation to zero of the radiance decrease versus the Sun-to-Venus distance allows estimating the solar radius at the time of first and fourth contacts. This method presents the advantage of being almost independent of the plate scale, the distortion, and the point spread function (PSF) of the imager. It has been applied to the two space solar imagers, PICARD - Solar Diameter Imager and Surface Mapper ${ }^{9}$ (PICARD-SODISM, see optical path in Figure 3) and Solar Dynamics Observatory - Helioseismic and Magnetic Imager (SDO-HMI), during the 2012 June 5-6 Venus transit. The results found, rounded to 959.85 arc-seconds for SODISM (very close to that obtained with the conventional method) and 959.90 arc-seconds for HMI. ${ }^{8}$ This is one of the first determination of the solar radius outside the atmosphere made by two different instruments.

From time immemorial, humanity has strived to measure the size of the Sun. Today, this challenge is about to come to its end. Thus, it is therefore necessary, after 120 years, to revise the value of the solar radius. A solar radius value of $696,156 \pm 145$ kilometres $(1 \sigma)$ can be adopted. This result is quite similar to that measured by Jean Picard, several centuries earlier.
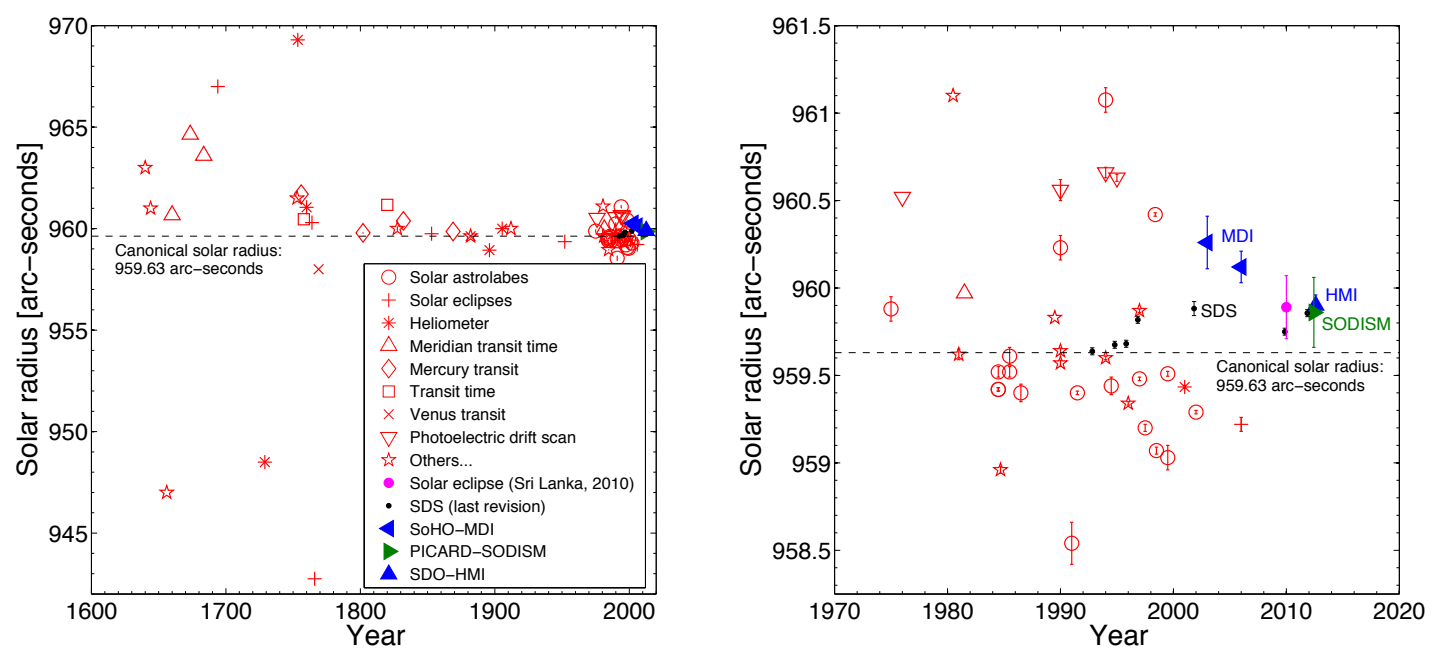

Figure 2. (left) Solar radius measurements (red symbols) made since the seventeenth century (see survey of historical measurements made by Rozelot and Damiani ${ }^{10}$ ). The mean value of all these measurements is close to 960 arc-seconds. (right) Focus on solar radius measurements made since 1970. Adassuriya et al. ${ }^{11}$ found a solar radius of $959.89 \pm 0.18$ arc-seconds (see magenta circle symbol) during solar eclipse in Sri Lanka (ground measurement). SDS balloon flight measurements ${ }^{7}$ are represented with black circles. SoHO-MDI and SDO-HMI records are represented with blue symbols (measurements obtained from space). PICARD-SODISM measurement obtained during the transit of Venus is represented with a green symbol (determination outside the atmosphere). 


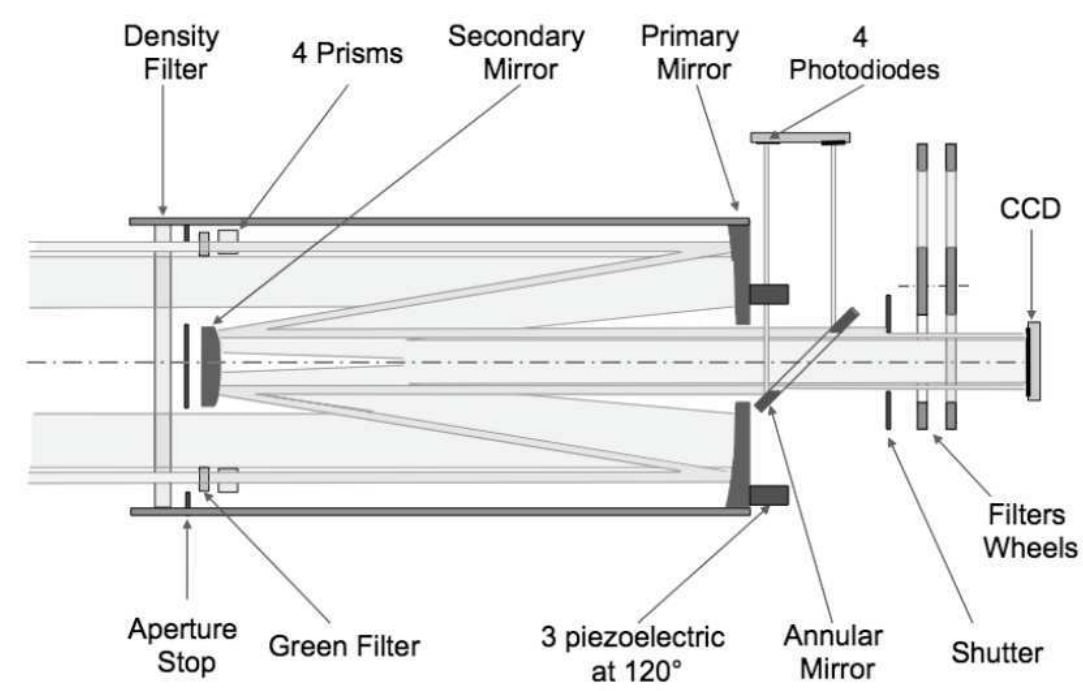

Figure 3. PICARD-SODISM main optical path consists essentially of a front window (density filter), a primary mirror (M1), a secondary mirror (M2), interchangeable interference filters, and a charge coupled device (CCD). The CCD detector array has $2048 \times 2048$ pixels of $13.5 \mu \mathrm{m}$ pitch. Focal length and CCD format lead to a field of view of $\sim 36 \times 36$ arc-minutes, and a plate scale of $\sim 1.06$ arc-seconds per pixel. The distance between M1 and M2 is $323.5 \mathrm{~mm}$, and the distance between $\mathrm{M} 2$ and the CCD is $516.8 \mathrm{~mm}$.

\section{TOTAL SOLAR IRRADIANCE}

Solar irradiance is the primary source of energy reaching the Earth-atmosphere system. The TSI value is crucial for solar physics and for the Sun-Earth connection. A previous period of low sunspot activity, the Maunder minimum, beginning in about 1645 and continuing until 1715, coincided with part of the Little Ice Age. This period of cooling that occurred after the Medieval Warm Period and affecting parts of the globe, corresponds to a drop in the TSI. Various studies show this phenomenon with different approaches and with very different results. Some analyses suggested that solar conditions during the recent solar minimum might give a good idea of solar conditions during the Maunder minimum, ${ }^{12}$ while other analyses suggest that TSI during the Maunder minimum was around $5.5 \mathrm{~W} . \mathrm{m}^{-2}$ below the last solar minimum. ${ }^{13}$ This difference is larger than other estimations published in the literature. ${ }^{14,15}$ However, solar irradiance measurements have only been available for the last three decades, and thus there is a need for the use of models over longer time scales. These TSI reconstructions rely, amongst others, on solar activity indices such as sunspot number, geomagnetic indices such as $a a$ index, cosmogenic isotopes such as carbon-14 and beryllium-10, or solar models such as flux transport models. In fact, the TSI is an important input for all climate models. Nevertheless, neither its direct nor its indirect influence explains global warming over the past century, ${ }^{16,17}$ and certainly not over the past 35 years (Sun and climate have been going in opposite directions), which limits the role of solar irradiance variation in twentieth century global warming. Recent research has added further complexity to the Sun-Earth relationship. ${ }^{18}$ The TSI during the Maunder minimum is a matter of debate as the absolute TSI value. From current technology and with the advent of the space age, the absolute TSI value can be determined with good accuracy. Claude Pouillet (1790-1868) made the first measurements of this fundamental astrophysical quantity ${ }^{19}$ between 1837 and 1838 . The first TSI estimate was $1228 \mathrm{~W} \cdot \mathrm{m}^{-2}$, very close to the current estimate. TSI measurements have been performed in orbit since 1978 and do not provide information on longer-term secular trends. TSI variations were too small to detect with the technology available before the spacecraft era. The instruments currently used in space yield a TSI variation that is substantially identical. Total solar output is now measured to vary (over the last three 11-year sunspot cycles) by approximately $\pm 0.05 \%$. The TSI is in phase with solar activity because the Sun is faculae-dominated, which is not the case for every Sun-like star. During a solar cycle, TSI variations are relatively small. Nevertheless, these changes have been proposed as a possible driver of climate change. ${ }^{20,21}$ Based on measurements collected from different space instruments over the past 35 years, the absolute value of TSI (representative value for a quiet Sun) has gradually decreased from $1371 \mathrm{~W} \cdot \mathrm{m}^{-2}$ in 1978 to around $1362 \mathrm{~W} . \mathrm{m}^{-2}$ in 2013 , mainly due to the 
radiometers calibration differences. Since 1978, a series of Active Cavity Radiometers (ACRIM) has gathered precise total solar irradiance data but global climate trends of interest occur over longer time spans. The Total Irradiance Monitor (TIM) of the Solar Radiation and Climate Experiment (SORCE) mission ${ }^{22}$ measured a TSI close to $1361 \mathrm{~W} \cdot \mathrm{m}^{-2}$. This was down about $4.5 \mathrm{~W} \cdot \mathrm{m}^{-2}$ compared to measurements made by other space radiometers in service at the same period. ${ }^{23}$ This measurement has been recently confirmed by two radiometers on board the PICARD space mission. ${ }^{4,24}$ In fact, the determination of TSI from Solar Variability PICARD (SOVAP) radiometer has required the establishment of a new instrumental equation. A new parameter has been integrated from a theoretical analysis, which highlights the thermo-electrical non-equivalence of the radiometric cavity. From this new approach, we obtain values that are lower than those previously provided with the same type of instruments. Based on the SOVAP data, we predict the TSI input at the top of the Earth's atmosphere at a distance of $1 \mathrm{AU}$ from the Sun to be $1362.1 \mathrm{~W} \cdot \mathrm{m}^{-2}$ with an uncertainty of $\pm 2.4 \mathrm{~W} \cdot \mathrm{m}^{-2}(1 \sigma)$. A TSI value around $1362 \mathrm{~W} . \mathrm{m}^{-2}$ may be proposed as a reference value (TSI-2008 reference). ${ }^{25}$ Thus, we can reconstruct the evolution of TSI over the past three decades from measurements made by the space radiometer (Figure 4). We notice that the values of the TSI during the solar minimum differ slightly depending on radiometers. A question that remains open: is that all the TSI solar minima are at the same level?

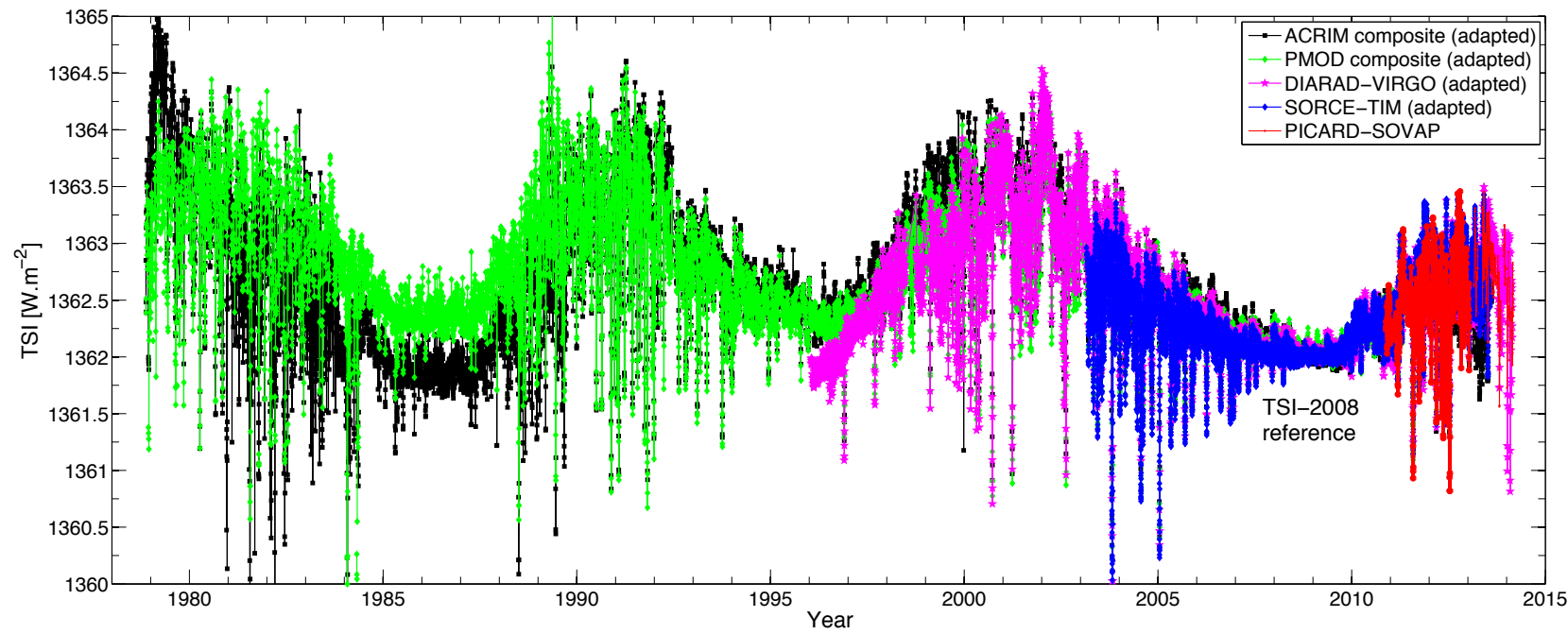

Figure 4. TSI record (composite), showing $\pm 0.05 \%$ variations with solar activity on 11-year. Adapted (or adjusted) ACRIM composite (black) with TSI-2008 reference, represents the longest series. Adapted (or adjusted) composite TSI time series (ACRIM and PMOD) highlighting differences for some solar minima. TSI variability time series for three space radiometers are plotted: Differential Absolute Radiometer - Variability of Irradiance and Gravity Oscillations (DIARAD-VIRGO), SORCE-TIM and PICARD-SOVAP.

Moreover, using reconstructions we can estimate the absolute value of the TSI during the Maunder minimum. If we take the model proposed by Krivova et al. ${ }^{14}$ as reference, we have a TSI close to $1361 \mathrm{~W} . \mathrm{m}^{-2}$ during this particular period. However, the difference between the TSI-2008 reference and the TSI value during the Maunder minimum is still open (Figure 6).

\section{SOLAR RADIUS VARIABILITY}

Possible temporal variations of the solar radius are important as an indicator of internal energy storage and as a mechanism for changes in the TSI. Long-term cyclic variations in the TSI are caused by the corresponding changes in the solar radius $\left(R_{\odot}\right)$ and effective temperature of the Sun $\left(T_{\text {eff }} \sim 5778 \mathrm{~K}\right)$, which represents another fundamental parameter of our star, where:

$$
\frac{\Delta T S I}{T S I}=2 \times \frac{\Delta R_{\odot}}{R_{\odot}}+4 \times \frac{\Delta T_{\text {eff }}}{T_{\text {eff }}}
$$




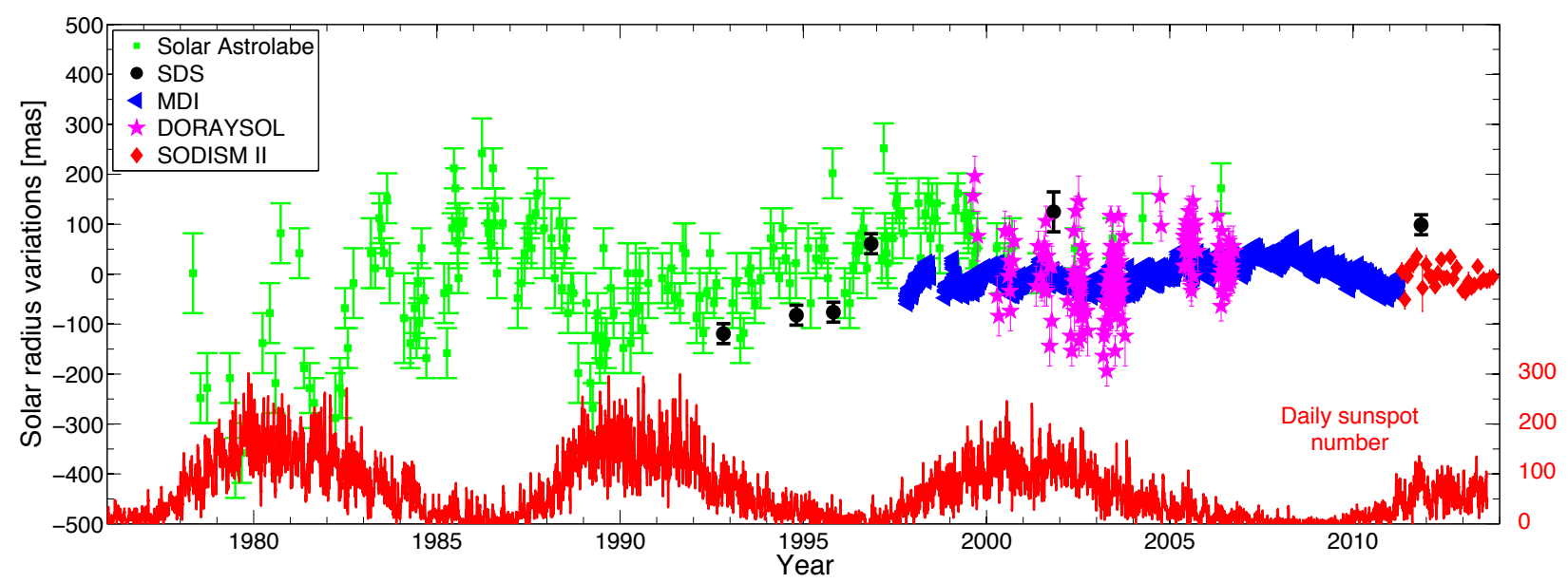

Figure 5. Evolution of the solar radius variations over time for ground instruments (Solar Astrolabe, DORAYSOL and SODISM II monthly mean at $782.2 \mathrm{~nm}$ ), balloon experiment (SDS) and space instrument (MDI) vs. daily sunspot number time series, which are correlated with TSI. For each series, the mean has been taken as reference value.

Thus, it is interesting to estimate the solar radius variations with the solar cycle. This information can provide data for the models to explain the TSI variations with the solar cycle $(\sim 0.1 \%)$. Assuming no variation in $T_{\text {eff }}$, the maximum possible variation $\Delta R_{\odot}$ of the solar radius cannot be more than 0.5 arc-second during a solar cycle. Among them, periodicities of 11-years (Schwabe solar cycle), 22-years (Hale cycle) and around one century (Gleissberg cycle) are likely to be related to solar activity, giving rise to discussion on the influence of solar variability on the Earth's climate. The relationship between the solar radius and the solar activity is the field of measurements and solar modelling. One of the first to raise questions with respect to the solar radius variations was Hermann Helmholtz (1821-1894). He had proposed a theory for the luminescence of the Sun. He stated that the Sun had been larger in the past and was slowly collapsing into itself due to its own gravity, releasing gravitational energy in the form of light and heat. But that was before nuclear fusion was discovered. Eddy and Boornazian were the pioneers in the field of measurement of solar radius variations, ${ }^{26}$ and in 1979 , they announced that their studies of solar measurement records showed that the Sun was shrinking (around 1 arc-second per century). Gilliland announced a secular decrease of around 0.1 arc-second per century. ${ }^{27}$ Other analyses concluded that there has been no detectable variation of the Sun over the past 250 years. ${ }^{28}$ Toulmonde made a compilation of all the solar radius measurements made between 1660 to 1995, and concluded that the mean solar radius at one astronomical unit is $960.0 \pm 0.1$ arc-seconds (value close to PICARD absolute measurement). His investigations did not reveal any substantial secular variation in the solar radius. ${ }^{2}$ However, it is very difficult to extract a trend in the solar radius from historical data. Thus, this problem is still a matter of debate. On the one hand, the variability of the solar radius can be analysed during a solar cycle. The series of measurements of the solar radius at Calern Observatory spans about 35 years (see Figure 5). The solar radius survey was initiated in 1975 with the Solar Astrolabe. Solar radius measurements performed at the Calern site have shown long-time variations. These variations reveal an anti-correlation with solar activity ${ }^{29}$ for the period 1975-1994. These results have raised many questions.

The SDS experiment confirms the solar radius variability through these seven flights covering the years 1992 to 2011. The solar radius is found to vary over that period by up to 0.2 arc-second. ${ }^{7}$ The variation is not in phase with the solar activity (Figure 5). An analysis of photometric images made at San Fernando Observatory from 1986 to 2004 shows a peak-to-peak solar radius change of 0.14 arc-second in phase with the solar cycle. ${ }^{30}$ Numerous studies of solar radius variations were carried out, but with conflicting results. On the other hands, data from the MDI instrument on the SoHo spacecraft show no evidence of secular trends in the solar radius, or variations attributable to the 11-year cycle at a level of a few milli-arc-seconds (mas). In addition, there is probably no link with solar activity. ${ }^{31}$ Systematic changes in the solar radius with the sunspot cycle must be smaller than 23 mas peak-to-peak ${ }^{32}$ (with the MDI instrument). Instrumental and atmospheric effects affect 


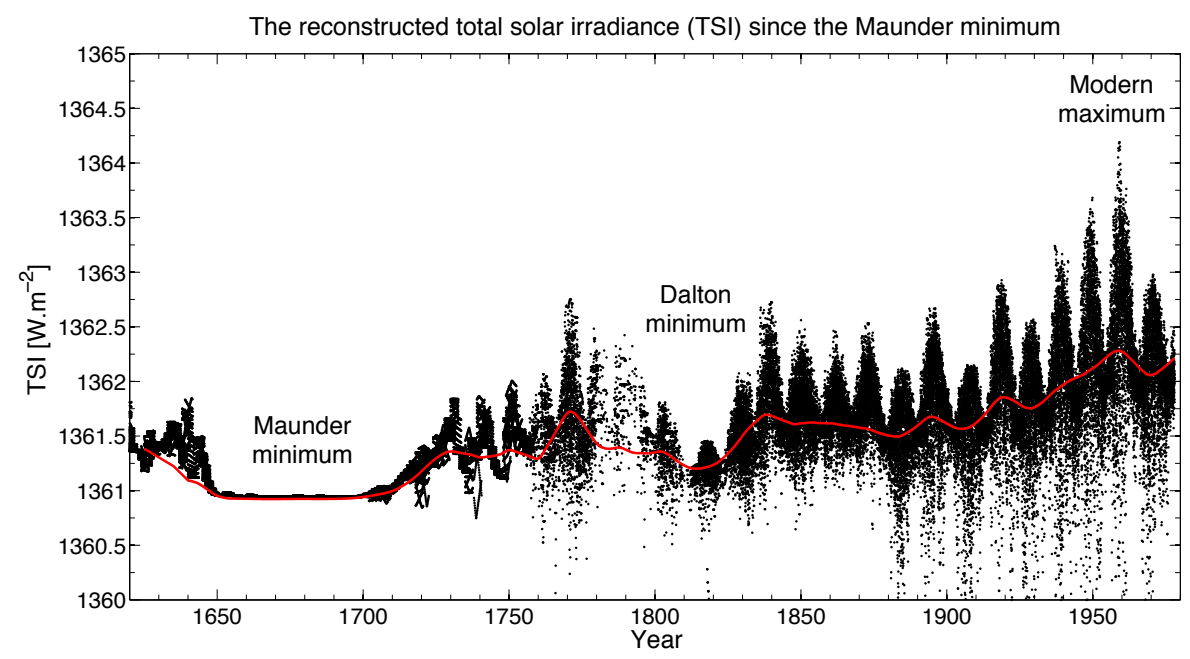

Figure 6. Reconstructed TSI (standard model based on the group sunspot number) since the Maunder minimum in black ${ }^{14}$ and reconstructed TSI during the Holocene period in red $^{15}$ using our TSI-2008 reference.

solar radius measurements. The Earth's atmosphere generates various hindrances that cause morphometric and photometric difficulties. They include refraction and turbulence, not to speak of scattering, extinction and diurnal alternation. It is suspected that the past inconsistencies regarding the temporal dependence of the solar radius measured from the ground would stem primarily from such contingencies. Ground solar astrometric variability can be accounted by long-term changes in the terrestrial atmosphere and balloon flights can be accounted by instrumental effects. Foukal et al. ${ }^{17}$ concluded that it is unlikely that solar radius measurements can reveal deeper lying sources of solar irradiance variations, as was originally hoped. ${ }^{16}$ Models of the solar surface reinforce this conclusion. The impact of simulated activity is a 14 mas shrinking of the solar layers near the optical depth unity. ${ }^{33}$ More than $85 \%$ of TSI variance is due to dark sunspots and bright faculae. Any residual TSI variations result from slower changes in the solar radius and or in convective patterns. Therefore, changes in the size of the solar disk contribute negligibly to the TSI during a solar cycle and probably during the last centuries. The current "PICARD SOL" ground-based measurements made at Calern Observatory, converge to the same conclusion as that derived from the analysis of data from the MDI instrument. In fact, the solar radius observed with "PICARD SOL" results from small variations after 36 months of measurements. The solar variability trend is less than 30 mas (Figure 5), and there is no direct link with the TSI. During a solar cycle, variation of the solar radius peak-to-peak is less than 20 kilometres. Variation of the solar radius since the minimum of Maunder is still open, but it is probably negligible. In fact, TSI varies by approximately $0.1 \%$ during a solar cycle and has changed by around $0.1 \%$ since the Maunder minimum, suggesting an increase of about $1 \mathrm{~W} \cdot \mathrm{m}^{-2}$ since 1700 for most recent reconstructions (see Figure 6), and in contrast to older reconstructions. This allows reinforcement of the weak solar radius variability during this period, according to Equation 1.

\section{SOLAR OBLATENESS}

The Sun is an almost perfect sphere. The rotation of the Sun involves a flatness of the polar regions. Its solar oblateness $\left(\Delta_{\odot}=\left(R_{e q}-R_{p o l}\right) / R_{p o l}\right)$, expressed as the equator-to-pole radius difference $(\Delta r)$, is about $10^{-5}$ of its radius. It is believed that the solar oblateness results from the internal rotation of the Sun and from its mass distribution. If the solar surface deformation provides an indirect information on the inner rotation profile and on the distribution of matter, it could also inform on the internal magnetic field or on the Earth-Sun relationship, so the magnitude of the solar oblateness stays an important ingredient to constrain models of the Sun interior and predictions of its variability. It also puts constraints on general relativity. However, due to its very small value, the solar oblateness is very difficult to measure and its determination requires a very good understanding of any possible instrumental effect. Based on measurements collected from various instruments over the last 140 years,${ }^{34}$ the measured solar equator-to-pole radius difference has generally become smaller over time from 500 mas 


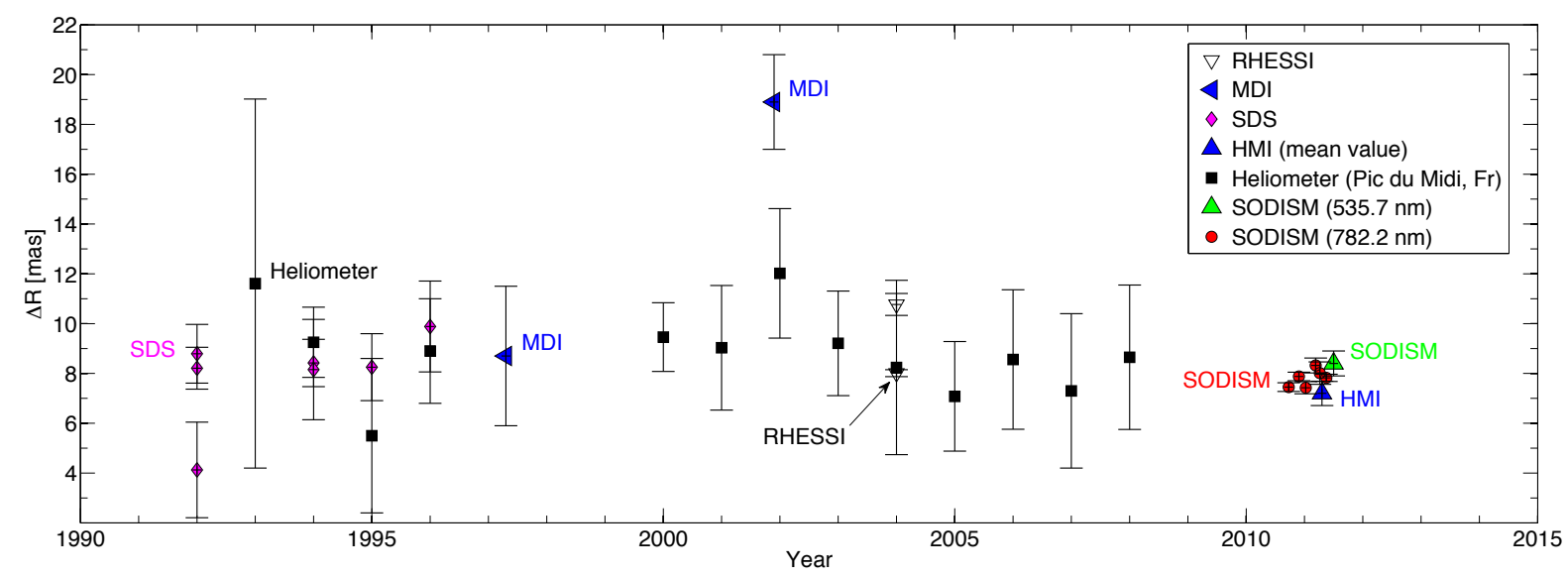

Figure 7. Solar equator-to-pole radius difference $(\Delta r)$ measured since 1992 from ground-based instruments such as Pic du Midi heliometer, instruments on board balloon such as SDS, and space instruments (MDI, RHESSI, HMI, and SODISM).

in $1870^{35}$ to 8.4 mas in $2014,{ }^{36}$ mainly due to the instrument precision and data process. Since the late nineteenth century, a large number of solar oblateness measurements has been made (with ground-based instruments such as Pic du Midi heliometer, ${ }^{34}$ instruments on board balloon such as SDS, ${ }^{37}$ and space instrument). Here we will examine solar oblateness measurements over the last two decades (see Figure 7), because there were too many disparities in the data before that. The first measurement of the solar oblateness obtained in space, was conducted with MDI instrument, where the solar equator-to-pole radius difference (in 1997) was $8.7 \pm 2.8$ mas. ${ }^{38}$ Since then, other space instruments have made this measurement: Reuven Ramaty High Energy Solar Spectroscopic Imager (RHESSI) with his instrument Solar Aspect Sensor $\left(8.01 \pm 0.14 \mathrm{mas}^{39}\right)$, HMI $\left(7.2 \pm 0.49 \mathrm{mas}^{40}\right)$, and SODISM (for two typical wavelengths: $535.7 \mathrm{~nm}$ and $782.2 \mathrm{~nm}$ ). PICARD-SODISM measurements of the solar shape during special roll maneuvers of the spacecraft have produced a precise determination of the solar oblateness. At $782.2 \mathrm{~nm}$, the PICARD-SODISM $\Delta r$ value is close to $7.9 \pm 0.4$ mas at one $\sigma$ (mean of the measurements obtained from September 2010 to May 2011) to take into account the systematic errors (Meftah et al., work in progress). Moreover, there does not appear to be any correlation with the total solar irradiance variations, considering that the solar oblateness variation, if it exists, is included in the margins of uncertainty. This remains consistent with the results obtained by SDO/HMI instrument. Another important measurement was made by the SODISM instrument in July 2011. At $535.7 \mathrm{~nm}$, the PICARD-SODISM $\Delta r$ value is close to $8.4 \pm 0.3 \mathrm{mas}^{36}$ at one $\sigma$. Thus, the most realistic solar equator-to-pole radius difference value is close to $8.1 \pm 0.6$ mas (from the mean value of the two PICARD wavelengths). Since the nineteenth century, scientific community has strived to measure the oblateness of the Sun. Today, this challenge is about to come to its end. Thus, a solar equator-to-pole radius difference value of $5.9 \pm 0.5$ kilometres $(1 \sigma)$ can be adopted. Indeed, PICARD reference value is compatible with all measurements made from space instruments during last years.

\section{CONCLUSION}

PICARD provides a distinctive opportunity to investigate absolute values of the solar radius, the total solar irradiance, the solar oblateness and their variations. Through the PICARD mission, we have developed new computational techniques to estimate the solar radius, the total solar irradiance and the solar oblateness. For the first time, simultaneous measurements were made by identical ground and space instruments. Based on the PICARD data, we believe that the photospheric solar radius is close to $696,156 \pm 145$ kilometres $(1 \sigma)$. During a solar cycle, variation of the solar radius peak-to-peak is less than 20 kilometres. Based on the PICARD data, we predict the TSI input at the top of the Earth's atmosphere at a distance of 1 AU from the Sun to be $1,362.1 \mathrm{~W} \cdot \mathrm{m}^{-2}$ with an uncertainty of $\pm 2.4 \mathrm{~W} \cdot \mathrm{m}^{-2}(1 \sigma)$. Total solar output varies by approximately $\pm 0.05 \%$, over the last three 11-year sunspot cycles and with a weak solar cycle 24. Based on PICARD data, we believe that the solar equator-to-pole radius difference value is close to $5.9 \pm 0.5$ kilometres $(1 \sigma)$. Moreover, there does not appear to be any correlation between the solar oblateness variations and the total solar irradiance variations. 


\section{ACKNOWLEDGMENTS}

This work was carried out at the LATMOS with the support of the CNES and the CNRS. We dedicated this work to the memory of Jean Picard and Claude Pouillet who inspired a generation of astrophysicists to consider the measurement and interpretation of these fundamental parameters of solar astrophysics.

\section{REFERENCES}

1. Ribes, E., Ribes, J. C., and Barthalot, R., "Evidence for a larger sun with a slower rotation during the seventeenth century," Nature 326, 52-55 (Mar. 1987).

2. Toulmonde, M., "The diameter of the Sun over the past three centuries.," Astron. Astrophys. 325, $1174-1178$ (Sept. 1997).

3. Auwers, A., "Der Sonnendurchmesser und der Venusdurchmesser nach den Beobachtungen an den Heliometern der deutschen Venus-Expeditionen," Astronomische Nachrichten 128, 361 (Dec. 1891).

4. Meftah, M., Hauchecorne, A., Crepel, M., Irbah, A., Corbard, T., Djafer, D., and Hochedez, J.-F., "The Plate Scale of the SODISM Instrument and the Determination of the Solar Radius at 607.1 nm," Solar Phys. 289, 1-10 (Jan. 2014).

5. Allen, C. W., [Astrophysical quantities] (1973).

6. Emilio, M., Kuhn, J. R., Bush, R. I., and Scholl, I. F., "Measuring the Solar Radius from Space during the 2003 and 2006 Mercury Transits," Astrophys. J. 750, 135 (May 2012).

7. Sofia, S., Girard, T. M., Sofia, U. J., Twigg, L., Heaps, W., and Thuillier, G., "Variation of the diameter of the Sun as measured by the Solar Disk Sextant (SDS)," Mon. Not. Roy. Astron. Soc. 436, 2151-2169 (Dec. 2013).

8. Hauchecorne, A., Meftah, M., Irbah, A., Couvidat, S., Bush, R., and Hochedez, J.-F., "Solar Radius Determination from Sodism/Picard and HMI/SDO Observations of the Decrease of the Spectral Solar Radiance during the 2012 June Venus Transit," Astrophys. J. 783, 127 (Mar. 2014).

9. Meftah, M., Hochedez, J.-F., Irbah, A., Hauchecorne, A., Boumier, P., Corbard, T., Turck-Chièze, S., Abbaki, S., Assus, P., Bertran, E., Bourget, P., Buisson, F., Chaigneau, M., Damé, L., Djafer, D., Dufour, C., Etcheto, P., Ferrero, P., Hersé, M., Marcovici, J.-P., Meissonnier, M., Morand, F., Poiet, G., Prado, J.-Y., Renaud, C., Rouanet, N., Rouzé, M., Salabert, D., and Vieau, A.-J., "Picard SODISM, a Space Telescope to Study the Sun from the Middle Ultraviolet to the Near Infrared," Solar Phys. 289, 1043-1076 (Mar. 2014).

10. Rozelot, J. P. and Damiani, C., "Rights and wrongs of the temporal solar radius variability," European Physical Journal H 37, 709-743 (Oct. 2012).

11. Adassuriya, J., Gunasekera, S., and Samarasinha, N., "Determination of the Solar Radius based on the Annular Solar Eclipse of 15 January 2010," Sun and Geosphere 6, 17-22 (Nov. 2011).

12. Schrijver, C. J., Livingston, W. C., Woods, T. N., and Mewaldt, R. A., "The minimal solar activity in 2008-2009 and its implications for long-term climate modeling," Geophys. Res. Lett. 38, 6701 (Mar. 2011).

13. Shapiro, A. I., Schmutz, W., Rozanov, E., Schoell, M., Haberreiter, M., Shapiro, A. V., and Nyeki, S., "A new approach to the long-term reconstruction of the solar irradiance leads to large historical solar forcing," Astron. Astrophys. 529, A67 (May 2011).

14. Krivova, N. A., Balmaceda, L., and Solanki, S. K., "Reconstruction of solar total irradiance since 1700 from the surface magnetic flux," Astron. Astrophys. 467, 335-346 (May 2007).

15. Vieira, L. E. A., Solanki, S. K., Krivova, N. A., and Usoskin, I., "Evolution of the solar irradiance during the Holocene," Astron. Astrophys. 531, A6 (July 2011).

16. Foukal, P. and Fröhlich, C. and Spruit, H. and Wigley, T. M. L. , "Variations in solar luminosity and their effect on the Earth's climate," Nature 443, 161-166 (Sept. 2006).

17. Foukal, P., "A New Look at Solar Irradiance Variation," Solar Phys. 279, 365-381 (Aug. 2012).

18. Haigh, J. D., Winning, A. R., Toumi, R., and Harder, J. W., "An influence of solar spectral variations on radiative forcing of climate," Nature 467, 696-699 (Oct. 2010).

19. Pouillet, C. S. M., [Memoire sur la chaleur solaire] (1838).

20. Willson, R. C. and Hudson, H. S., "The sun's luminosity over a complete solar cycle," Nature 351, $42-44$ (May 1991).

21. Fröhlich, C. and Lean, J., "The Sun's total irradiance: Cycles, trends and related climate change uncertainties since 1976," Geophys. Res. Lett. 25, 4377-4380 (Dec. 1998).

22. Kopp, G. and Lean, J. L., "A new, lower value of total solar irradiance: Evidence and climate significance," Geophys. Res. Lett. 38, 1706 (Jan. 2011).

23. Dewitte, S., Crommelynck, D., and Joukoff, A., "Total solar irradiance observations from DIARAD/VIRGO," Journal of Geophysical Research (Space Physics) 109, 2102 (Feb. 2004).

24. Schmutz, W., Fehlmann, A., Finsterle, W., Kopp, G., and Thuillier, G., "Total solar irradiance measurements with PREMOS/PICARD," AIP Conference Proceedings 1531(1), 624-627 (2013). 
25. Meftah, M., Dewitte, S., Irbah, A., Chevalier, A., Conscience, C., Crommelynck, D., Janssen, E., and Mekaoui, S., "Sovap/picard, a spaceborne radiometer to measure the total solar irradiance," Solar Phys. 289, 1885-1899 (May 2014).

26. Eddy, J. A. and Boornazian, A. A., "Secular Decrease in the Solar Diameter, 1863-1953.," in [Bulletin of the American Astronomical Society], Bulletin of the American Astronomical Society 11, 437 (Mar. 1979).

27. Gilliland, R. L., "Solar radius variations over the past 265 years," Astrophys. J. 248, 1144-1155 (Sept. 1981).

28. Parkinson, J. H., Morrison, L. V., and Stephenson, F. R., "The constancy of the solar diameter over the past 250 years," Nature 288, 548-551 (Dec. 1980).

29. Laclare, F., Delmas, C., Coin, J. P., and Irbah, A., "Measurements and Variations of the Solar Diameter," Solar Phys. 166, 211-229 (July 1996).

30. Chapman, G. A., Dobias, J. J., and Walton, S. R., "On the Variability of the Apparent Solar Radius," Astrophys. J. 681, 1698-1702 (July 2008).

31. Kuhn, J. R., Bush, R. I., Emilio, M., and Scherrer, P. H., "On the Constancy of the Solar Diameter. II.," Astrophys. J. 613, 1241-1252 (Oct. 2004).

32. Bush, R. I., Emilio, M., and Kuhn, J. R., "On the Constancy of the Solar Radius. III.," Astrophys. J. 716, 1381-1385 (June 2010).

33. Piau, L., Collet, R., Stein, R. F., Trampedach, R., Morel, P., and Turck-Chieze, S., "Models of solar surface dynamics: impact on eigenfrequencies and radius," ArXiv e-prints (Sept. 2013).

34. Rozelot, J.-P. and Damiani, C., "History of solar oblateness measurements and interpretation," European Physical Journal H 36, 407-436 (Nov. 2011).

35. Poor, C. L., "The Figure of the Sun," Astrophys. J. 22, 103 (Sept. 1905).

36. Irbah, A., Meftah, M., Hauchecorne, A., Djafer, D., Corbard, T., Bocquier, M., and Momar Cisse, E., "New Space Value of the Solar Oblateness Obtained with PICARD," Astrophys. J. 785, 89 (Apr. 2014).

37. Egidi, A., Caccin, B., Sofia, S., Heaps, W., Hoegy, W., and Twigg, L., "High-Precision Measurements of the Solar Diameter and Oblateness by the Solar Disk Sextant (SDS) Experiment," Solar Phys. 235, 407-418 (May 2006).

38. Emilio, M., Bush, R. I., Kuhn, J., and Scherrer, P., "A Changing Solar Shape," Astrophys. J. Lett. 660, L161-L163 (May 2007).

39. Fivian, M. D., Hudson, H. S., Lin, R. P., and Zahid, H. J., "A Large Excess in Apparent Solar Oblateness Due to Surface Magnetism," Science 322, 560- (Oct. 2008).

40. Kuhn, J. R., Bush, R., Emilio, M., and Scholl, I. F., "The Precise Solar Shape and Its Variability," Science 337, 1638- (Sept. 2012). 